\title{
Service Value Networks for Competency-Driven Educational Services: A Case Study
}

\author{
Iván S. Razo-Zapata, Pieter De Leenheer, Jaap Gordijn, and Hans Akkermans \\ VU University, Amsterdam, The Netherlands \\ \{i.s.razozapata,p.g.m.de.leenheer,j.gordijn\}@vu.nl
}

\begin{abstract}
Service networks represent a flexible way for delivering services to customers. In earlier work, we have applied the $e^{3}$-value methodology to conceptually model such networks. This paper, however, presents an approach for composing Service Value Networks (SVNs) based on customer and supplier perspectives. A broker is in charge of composing a SVN that reflects not only customer desires but also supplier offerings. Moreover, the application of using real-world services is shown by means of a case study. Finally, we provide some reflections as well as future lines of research.
\end{abstract}

\section{Introduction}

Service industry has been experiencing an enormous growing in the last years, it encapsulates over $70 \%$ of USA and Europe economies. Although the evident potential of this sector is clear, there are still some misunderstandings in areas like service composition, customer targeting and service provision [14]. Service composition briefly states that, given a specific customer need, at least one arrangement of service suppliers must be automatically composed to cope with such a need [9]. The assumption here is that due to the wideness of customer needs, it is necessary to combine the functionality of several services to cope with such needs. Even though service networks represent a flexible and dynamic way for service delivering, yet some knowledge gaps exist, specially regarding strategic bundling of services and value (co)creation within the network.

In this paper we aim at a framework for SVN composition from a business-oriented perspective. By business orientation we mean that the framework must take into account economic relationships rather than work flow properties. Business models are centred around the notion of value, therefore it is relevant to determine who is offering what of value to whom and what expects of value in return [10].

We propose an interactive dialogue to express customer needs based on marketing theory [15]. In addition, we also provide capabilities to publish service offerings by means of an ontology-based catalogue. Moreover, since mass configuration of products is playing an important role, dynamic composition of SVNs has been also supported. Finally, our long-term ultimate goal is to automatically compose a SVN, including the required business processes and Information Technology (IT) support in the form of web services. Such IT is then aligned with the business, since both are designed in an integrated way.

The rest of the paper is organized as follows. Sect. 2 introduces a set of basic concepts. In Sect. 3 we describe some related work. Afterwards, Sect. 4 provides the 
description of our case study. Later on, Sect. 5 presents our approach for SVN composition while Sect.6 shows the performance of such approach in our case study. Finally, conclusions and future work are described in Sect.7.

\section{Basic Concepts}

Service has become a term loaded with different meanings in different circumstances, mostly depending on who uses it [48]. In this paper, we focus only on a set of features that are relevant for our research. We refer to a service as follows.

Definition 1. A service is an economic activity possessing intangible nature and offering consequences for which customers are willing to sacrifice 481 .

Moreover, we are interested in services that can be provided through IT infrastructures, e.g. internet connectivity, network hosting amongst others. Such services are commonly offered by alternative suppliers which can publish their offerings with different consequences. In addition, sometimes customer requirements can not be covered by a single service but by a combination of them, which can involve one or more suppliers i.e. a service bundle.

Definition 2. A service bundle is a package of one or more services, these services can be provided by a single entity or by different enterprises, who each focus on their core competency [8].

When bundles or single services are offered to final customers we say that there is a business to customer (B2C) relationship, otherwise, if offered to another service supplier we have a business to business (B2B) relationship. Furthermore, when suppliers work together to cover a specific customer need, they compose a service network. The creation of such networks is, most of the time, driven by the value they provide to customers. Value networks have been broadly defined by several authors, some of them use terms such as economic web, business web, value web or value constellation [15]. From these definitions we can abstract basic denominators. First of all, value networks are composed of economic entities. These entities work together to achieve some objective. In this way, we can consider that those entities can be represented by services.

Definition 3. A service value network is a flexible and dynamic delivery of services, such that a value-adding specific solution is delivered to the individual customen.

By means of SVNs it is possible to model not only the structure and dynamics of service networks but also customer expectations and their influence in these networks [5]. Fig.1] depicts the three types of actors participating in a SVN, as well as the interactions among them. On the one hand, a customer interacts with service suppliers by means of $\mathrm{B} 2 \mathrm{C}$ relationships. On the other hand, through $\mathrm{B} 2 \mathrm{~B}$ relationships, service suppliers not only interact with other service suppliers but also with service enablers.

\footnotetext{
${ }^{1}$ The sacrifice is usually expressed in terms of money exchange.

${ }^{2}$ Adapted from Hamilton, 2004 [14].
} 


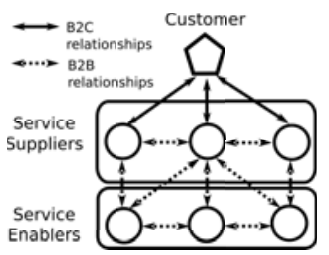

Fig. 1. Service Value Network

Customers are considered the starting point and the reason why a SVN must be generated. Briefly, according to their needs, customers send requests to the service network. Service suppliers provide resources that match customers requests. To foresee customer needs and adapt to changes in the network, they perform B2C and B2B relationships. Service enablers support the operation of service suppliers. In this sense, service enablers also establish B2B relationships with service suppliers.

Furthermore, there are at least two aspects pushing up the need for service value networks: 1) policy issues: due to market liberalization, many services that were traditionally provided by monopolies can be now offered by alternative suppliers. 2) rapid technological change: many sectors, like the music industry, are experimenting revolutionary changes in their traditional business models, mainly because of the emergence of new ways of delivering services [19].

In the end, we aim at a runtime composition framework where customers and suppliers dynamically establish relationships with each other. To achieve this goal, we propose the use of customer and supplier ontologies where concepts at both sides can be automatically matched allowing for the establishment of B2C and B2B relationships. Moreover, we are interested in modelling business-oriented aspects such as value exchanges.

\section{Related Work}

Since service network composition must deal with issues related to establishing B2C and $\mathrm{B} 2 \mathrm{~B}$ relationships, we have analyzed related work about service composition and service network modelling. Moreover, we have also made a distinction between $e^{3}$ value based and service science approaches.

Although many $e^{3}$-value inspired approaches focus on business aspects, they have a static flavor. According to Dustdar et al., [9], static approaches perform several step during design-time which make them unsuitable for dynamic composition. Zlatev, [24], proposes a framework for service composition based on value, process and goal models, however the transformations among these three representations require a lot of manual steps, which is not appropriate for automation. VBC, [18], aims at an automatic framework. Although the idea about using a value meta-model to compose service networks is well supported, the lack of results brings about some doubts over its applicability. $e^{3}$ service , [8], is one of the latest efforts in the field of value modelling. De Kinderen et al., propose a framework for matching customer and supplier views. Even though, $e^{3}$ service allows B2C interaction for service bundling, the main drawback is that the 
service bundles have been generated at design time. Serviguration [4] presents a more dynamic strategy for service bundling, nevertheless, the generated bundles do not always depict the concept of economic reciprocity, i.e. what they expect in return for an object of value delivered, which is important to understand the value exchanges within a SVN.

Traverso and Pistore, [23], have proposed a framework which makes use of a Model Based Planner (MBP). Because the MBP module generates work-flow-based plans, this approach is not business oriented and does not support any visualization for the generated plans. OntoMat-Service, [2], offers a dynamic approach for service composition. Although the approach allows basic modelling and visualization of service networks, it is focused on process-oriented issues. METEOR-S, [22], provides Semantic Process Templates (SPTs) to discover and add services. Because SPTs model the required activities to be performed, they give a basic notion of how a service network can be described. However, because of SPTs construction is performed at design-time, METEOR-S is an static approach. Moreover, it is also process-oriented. DynamiCoS, [21], supports run-time discover and composition of services. Nevertheless, it does not offer any formal definition to represent service networks in terms of economical exchanges. Finally, Danylevych et al., [6], present a framework for modelling service networks. Even though it gives formal backgrounds, some manual steps are required to build the service network and to transform it into BPMN.

As can be observed, many service science approaches focus on process-oriented issues, that is why the composition process is usually modelled as a planning problem. However, as we describe in Sect. 5, a business-oriented approach might rely on other strategies. Finally, we emphasize our interest in dynamic and business-oriented approaches allowing for customers and suppliers to co-create service value networks as well as providing visual representation of economic relationships among them.

\section{Case Study}

The European employment market is characterized by a contradictory situation: a very large number of candidates fail to find a job; and many employers are unsuccessful in locating appropriate candidates for their vocations. Given a Vocational Competency Ontology (VCO) (collaboratively developed in previous work [7]), skill gap analysis can overcome the semantic mismatch between candidate and market profiles, and capture a candidate's missing competencies. Stakeholders include educational institutes, public employment organizations, and industry partners from different European countries.

Central actor driving the evolution of the Educational Service Web are the enterprise ecosystems. Once set out the goals and strategy of the company, different supporting business processes are lined out, involving the creation of (new) functions and tasks. Each of them require human performance, which in turn require certain competencies. From this feedback loop, relations between Functions and competencies emerge. To describe competencies, there is a widely used HR-XML-standard called reusable competency definitions (RCDs). RCDs are yet underspecified so they retain their generative character. To describe the relationship between functions and competencies enterprises define function profiles, which usually contain a competency map, RCDs and 


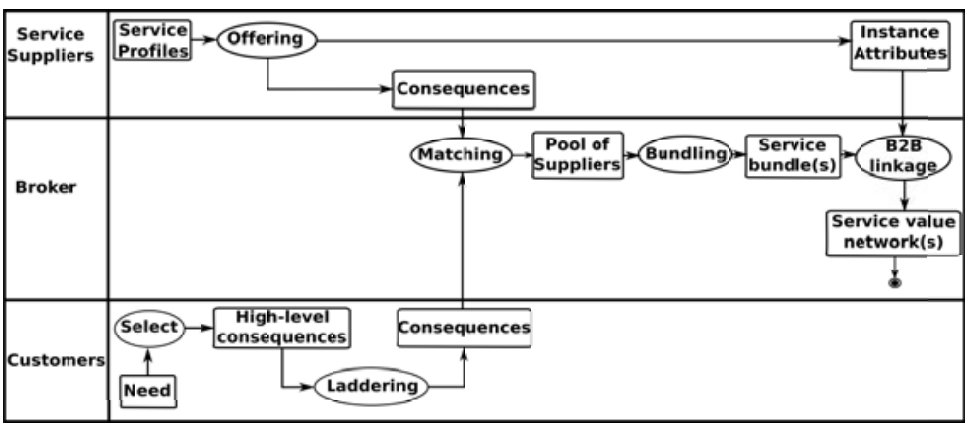

Fig. 2. Service Value Network composition

proficiency levels for Competencies (default +1) (IEEE score or SCORM uses an interval of -1 to +1$)$.

E.g., in the automotive industry functions are categorized along the car manufacturing process: going from press shop, to the body shop, to finally end at the assembly shop 3 . In order to perform each of those functions, human operators with specialized competencies are required.

The candidate's search is equally driven by populating its CV by RCDs he collects through experience and education. If its current CV shows gaps to fulfil a certain function profile, a need emerges that has to be answered by the Service Web. The final stakeholder's cycle are the educational service suppliers. They monitor needs and function profiles and define services accordingly.

Currently the stakeholder community is simplified for the sake of illustration. In reality there are additional parties that are responsible for identifying large gaps in the candidate pools and predicting future needs in education, and finally organize this education. The main point we want to make here is that all these parties can act independently and by doing so converge towards each other as long as their information (about functions, competencies and education service offerings) is published using (as shown below) open (IEEE and HR-XML) standards like RCD, SCORM, and LOM.

\section{SVNs Composition}

In order to deal with composition of SVNs a novel approach based on several steps is proposed in Figure 2. The idea is to provide a framework for achieving automatic composition of SVNs. Such framework relies on two perspectives, one for customer and the other one for suppliers. Initially, by means of a laddering process, a customer express its need in terms of consequences. Later on, a broker starts an automatic composition for matching, bundling and linking services. At the end of the process, a SVN with detailed information about specific suppliers and value exchanges must be generated. Subsections 5.1.5.3 give a deeper explanation of each step.

${ }^{3} \mathrm{cf}$. http: / / www. nedcar.nl/content/view/44/49/lang, en/ 


\subsection{Supplier Perspective}

To publish service offerings we promote the use of an ontology. Fig. 3 depicts an ontology, which is the result of aligning the $e^{3}$ service [8] supplier ontology with the $e^{3}$-value [11] ontology. We only describe what is relevant for defining supplier profiles. Service suppliers are actors performing activities (value activities) to produce resources (value objects) which can be offered to customers. In addition, as a result of its use or consumption, a value object has functional consequences and quality consequences. Finally, a service bundle consists of service elements which are special types of value activities. For a full elaboration of these concepts we refer to [11] and [8].

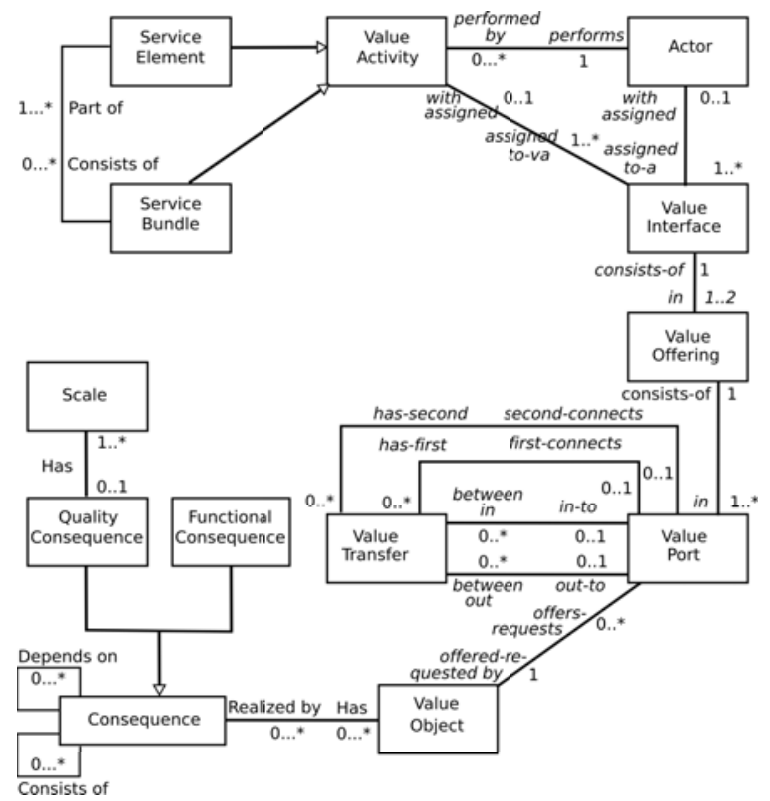

Fig. 3. The $e^{3}$ service [8] supplier perspective ontology aligned to the $e^{3}$-value [11] ontology

\subsection{Customer perspective}

In the same way, customers can express their needs by using another ontology. Figure 4 shows a UML rendering of the customer perspective. It is based on concepts from established customer needs literature [815].

- A Need represents a problem statement or goal (see[3[15]8] 4 . E.g., a job candidate has a need to fulfil a certain Function profile.

- A Consequence is anything that results from consuming (a combination of) valuable service properties [13 8]. We distinguish between two types.

\footnotetext{
${ }^{4}$ Needs are the basic human requirements. Wants are specific objects that might satisfy the need.
} 


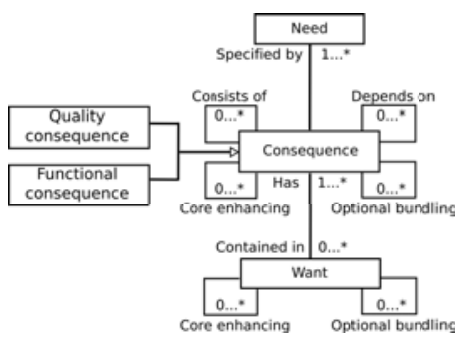

Fig. 4. The $e^{3}$ service customer perspective ontology, based on [8]

- Functional Consequence [8] represents the functional goal that can be achieved through consumption of a service that has a certain valuable property. E.g., a Functional Consequence from the need to fulfil a certain Function profile are all the involved competencies5. E.g., the consequence "read and write" consists of the consequences "read" and "write"; or the consequence "writing english" is core-enhancing for "programming", etc..

- Quality Consequence [8] A quality consequence expresses qualitative properties of other. Because it expresses the qualitative properties of another Consequence, a Quality Consequence cannot be acquired separately: It always depends on (a relation between Consequences ) another type of Consequence. E.g., Writing English has a required proficiency as a Quality Consequence.

- A Want [8] is a solution that is commercially feasible to be provisioned on its own. As a Want indicates a solution available in the market, at least one supplier should be willing to provide the solution. An example of a Want could be learning objects for which multiple institutes are accredited to deliver them.

- A Scale [8] groups Quality Consequences of the same type. E.g., a proficiency level according to IEEE or SCORM is defined by a number in an interval between -1 and +1 .

\subsection{Combining Perspectives}

To compose a SVN according to customer-expressed needs, some steps must be performed: offering, laddering, matching, bundling, and B2B linkage.

Offering suppliers publish their resources by using service profiles, which are designed according to the ontology in Fig. 3. Moreover, these profiles also enable interaction not only with customers but also with other suppliers and enablers.

Laddering is a marketing practice which uses a conceptual map to represent how a customer links specific product attributes to high-level values [8]. In our case we apply it to link service consequences to customer needs via high-level consequences (Fig. 4).

\footnotetext{
${ }^{5}$ The structure of the tree in between function and competencies may be defined by the RCM as described above.
} 
Matching determines a pool of service suppliers that plausibly provide part of the required consequences. Due to the variability of customer needs, single suppliers rarely provide all the required consequences on their own. Consequences are the key components for matching the two perspectives. For each functional consequence at the customer side, this matching process performs a (semantic) comparison with all the functional consequences expressed at the supplier side and retrieves the service that offers the required consequences.

Bundling takes as input the pool of suppliers and finds combinations of suppliers that collectively cover the required consequences. At this stage, we have applied a modified version of Baida's algorithm [4]. Whereas the original Baida's algorithm bundles services according to the value objects they offer, we bundle services depending on the consequences they provide. Moreover, our algorithm generates bundles depicting the concept of economic reciprocity by means of $e^{3}$-value interfaces and ports. A deep explanation of such algorithm is out of the scope.

B2B Linkage is applied to those bundles requiring to exchange resources with service enablers. For instance, for educational services such as On-line IT Training, enabler services like Black Board or Simulation platforms might be needed. Therefore, those additional services must be obtained by exchanging resources between service suppliers and service enablers. Moreover, at this stage, instance attributes of services are used to perform the linkage 6 . In previous work, [12] , we have defined a methodology for achieving B2B linking. Such methodology involves a searching process based on the value objects a service supplier requires.

As can be observed in Fig. 2, once the pool of suppliers has been found, the composition of the SVN involves bundling and B2B Linkage. We claim that by firstly generating service bundles, the broker can focus on the main services to fulfil the customer need. At this point strategic decisions might take place. On the contrary, B2B linkage requires less strategic consideration and constraints might be relaxed. However this step is also important to have a complete SVN and compute the total cost of what is offered to the customer.

\section{Running Example}

In order to show the applicability of our composition approach, we have developed a Java-and-Jena-based 7 prototype which automates the composition of a SVN to fulfill a candidate's need8. The educational e-service Web acts on publicly available instance data about related needs and services we found on the Web.

Offering. For modelling service suppliers and enablers, we surveyed a number of publicly available competency databases and harvested the National Database of Accredited Qualifications 9 (NDAQ). Fig.5]depicts a sample of the service catalogue for educational

\footnotetext{
${ }^{6}$ Instance attributes are details about resources such as price or resource's ids.

${ }^{7} \mathrm{http} / / /$ jena.sourceforge.net/

${ }^{8}$ We assume that such need was already identified during skill gap analysis.

9 http://www.accreditedqualifications.org.uk
} 


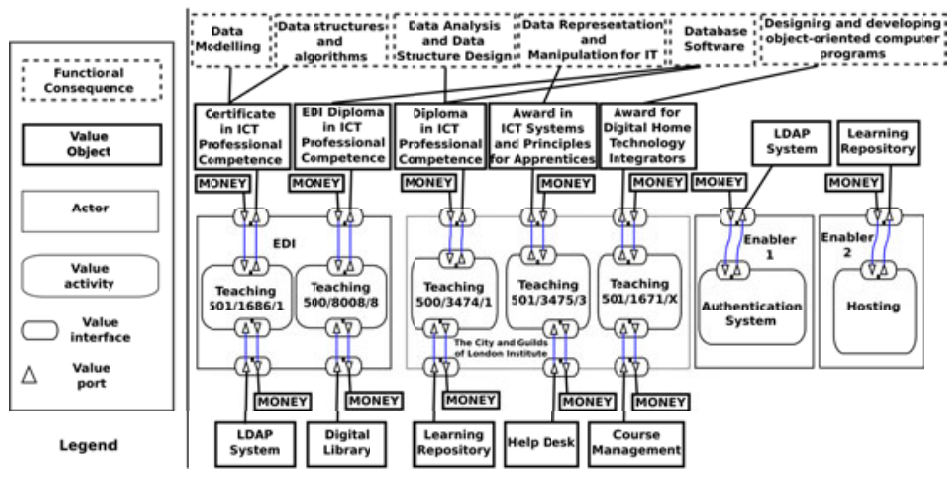

Fig. 5. Service catalogue generated from Web data in NDAQ

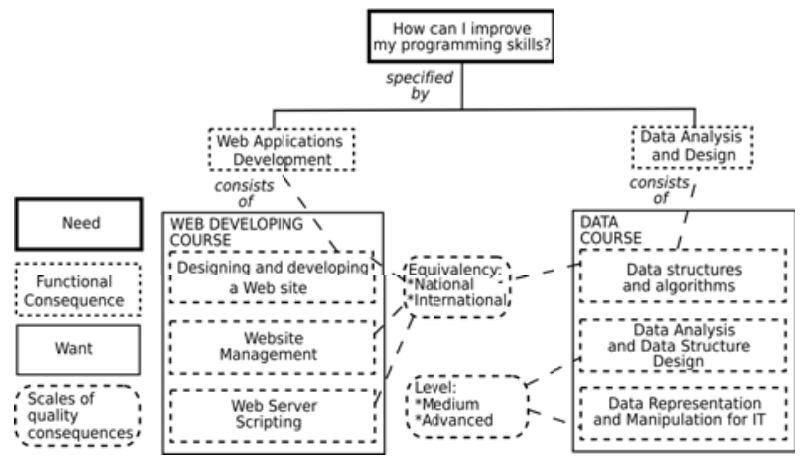

Fig. 6. Customer catalogue

services. These service profiles were designed based on the ontology depicted in Fig. 3 Service suppliers are represented by actors providing educational services (value activities) which in turn offer several resources (value objects) that have different functional consequences (Competencies). Service enablers are represented by actors providing only supporting services.

Laddering. At this step customers express their needs using an interactive dialogue in which they can refine vague needs in terms of functional consequences. To illustrate the laddering, we have designed a customer catalogue (Fig. 6) based on the NDAQ database, by using the ontology in Fig. 4 and grouping consequences according to possible courses in which they can be offered. Later on, these consequences are linked to customer needs via high-level consequences. The high-level customer need How can I improve my programming skills? is refined by two optional consequences: Web Applications Development and Data Analysis and Design. Theseconsequences are recursively refined further. E.g., if the consequence Data Analysis and Design is selected to cover the customer need, the laddering will determine that 


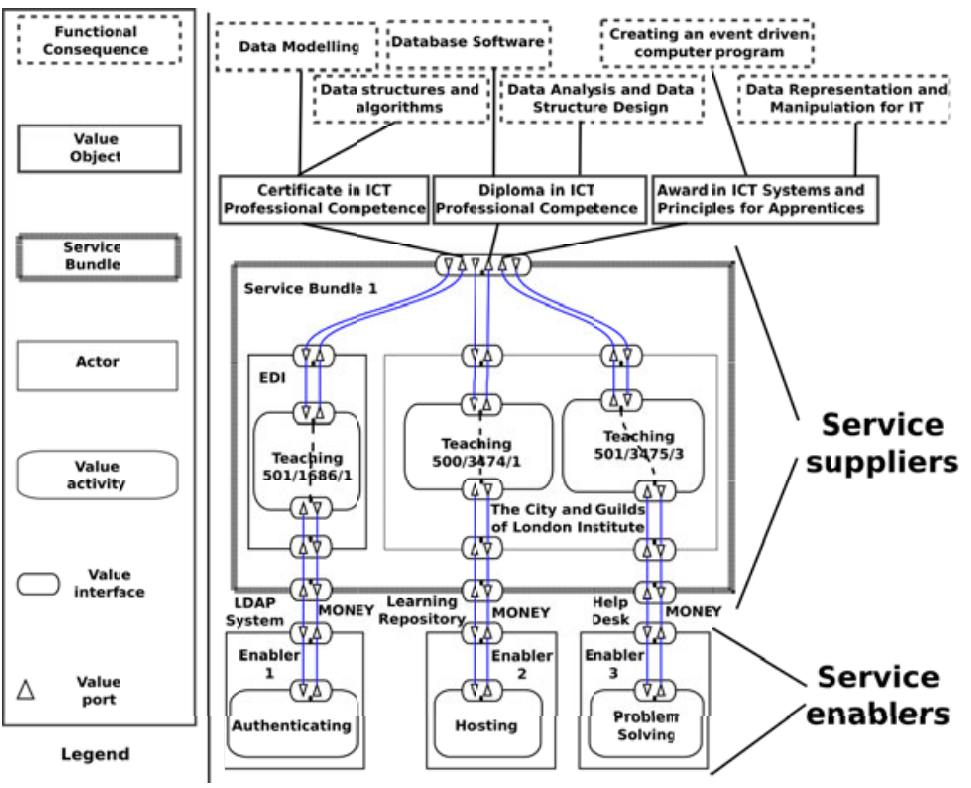

Fig. 7. Service Value Network to be offered at the Customer

the functional consequence Data structures and algorithms is more concrete to fulfil such need, since the first one consists of the second one.

The next step involves discovering more consequences through the notion of wants. In Fig. 6 the functional consequence Data structures and algorithms is contained in the Data Course want. By exploring this want, the functional consequences Data Analysis and Data Structure Design and Data Representation and Manipulation for IT are discovered. Finally, as part of the interactive dialog, customers are asked to evaluate functional consequences by scoring their quality consequences. E.g., for the functional consequence Data Representation and Manipulation for IT, a customer is requested to score the relevance of the functional qualities Medium and Advanced. In this way, unimportant functional consequences can be depreciated while preference for relevant functional consequences can be highlighted.

Matching. Once the required consequences have been specified, the matching process retrieves all the possible service suppliers that offer the required consequences. As already explained, the core of this process is a (semantic) comparison between concepts at both catalogues (supplier and customer).

Bundling. Fig. 7 depicts one of the alternative service bundles providing the required functional consequences for the customer need How can I improve my programming skills?. Since our algorithm generates several bundles, the depicted bundle was selected by hand. As can be observed, the bundle includes different suppliers which 
offer different services through a common interface that can be later offered to the final customer. Moreover, our bundle is visualized according to the $e^{3}$-value ontology.

B2B Linkage. Finally, once the bundles are generated, the last step is to solve the dependencies of the service suppliers. By dependencies we mean the extra resources that are needed by the service suppliers. Usually those extra resources might be obtained via other suppliers or enablers. In our case, as depicted in Fig. 7 , the service bundle gets the extra resources from a pool of service enablers. For instance, the service Teaching 500/3474/1 requires a Learning Repository which is obtained from the Hosting service that is provided by Enabler 2.

\section{Conclusions and Future Work}

In this paper we have presented a novel approach for automatically composing SVNs, which takes into account service suppliers and customers. On the one hand, our approach provides mechanisms for publishing supplier's offerings that can be later used to cover customer needs. On the other hand, it also provides a reasoning process, called laddering, that allows customer to represent vague needs in terms of specific consequences, which can be later matched with supplier's offerings. Since it is not only aligned with marketing theory concepts but also provides a better understanding about customer needs, the laddering is one of the benefits of this approach.

Once the matching is done, a bundling process generates all the possible bundles that can cover the given customer need. The last step is to solve all the bundle's dependencies, i.e. getting the extra resources that are required by each bundle (B2B Linkage). As a matter of fact, this dynamic on-the-fly composition of SVNs based on service bundling and B2B Linkage is other benefit of the approach. Moreover, by allowing to customers interacting with suppliers, we are not only providing a mechanism for cocreating new SVNs, but we are also increasing the perceived value of the SVN, since it fits better the customer need.

Although SVN composition is driven by a specific customer need, our approach offers a wide pool of SVNs to cover with such need. Consequently, the next steps in our research are focused on issues such as improvement of the bundling process, including business rules and selection of SVNs by customers.

To deal with the first issue we might explore combinatorial optimization strategies or even evolution-based methods. About business rules inclusion, we plan to apply them in both steps: in the bundling process and in the B2B linkage. Afterwards, to allow selection among alternative SVNs we will provide a mechanism to customers so they can explore all the SVNs and pick one according to her/his desires.

\section{References}

1. Allee, V.: A value network approach for modeling and measuring intangibles. In: Transparent Enterprise Conference (2002)

2. Agarwal, S., Handschuh, S., Staab, S.: Annotation, composition and invocation of semantic web services. Journal of Web Semantics 33(1), 1-24 (2004) 
3. Arndt, J.: How broad should the marketing concept be? Journal of Marketing 42(1), 101-103 (1978)

4. Baida, Z.: Software-aided service bundling. PhD thesis, Free University Amsterdam (2006)

5. Basole, R.C., Rouse, W.B.: Complexity of service value networks: conceptualization and empirical investigation. IBM Syst. J. 47, 53-70 (2008)

6. Danylevych, O., Karastoyanova, D., Leymann, F.: Service Networks Modelling: An SOA \& BPM Standpoint. J. UCS 38(5), 1668-1693 (2010)

7. De Leenheer, P., Christiaens, S., Meersman, R.: Business semantics management: a case study for competency-centric HRM. Computers In Industry 61(8), 760-775 (2010)

8. de Kinderen, S.: Needs-driven service bundling in a multi-supplier setting: The computational e3service approach. PhD Thesis, Free University Amsterdam (2010)

9. Dustdar, S., Schreiner, W.: A survey on web services composition. Int. J. Web Grid Serv. 1(1), $1-30$ (2005)

10. Gordijn, J., Akkermans, H., van Vliet, H.: Business Modelling Is Not Process Modelling. In: Mayr, H.C., Liddle, S.W., Thalheim, B. (eds.) ER Workshops 2000. LNCS, vol. 1921, pp. 40-51. Springer, Heidelberg (2000)

11. Gordijn, J., Akkermans, J.M.: e3-value: Design and evaluation of e-business models. IEEE Intelligent Systems, 11-17 (2001)

12. Gordijn, J., De, P.L., Razo-Zapata, I.S.: Generating service valuewebs by hierarchical configuration: An IPR case. In: HICSS 44 (2011)

13. Gutman, J., Reynolds, T.J.: Laddering theory-analysis and interpretation. Journal of Advertising Research 28(1), 11 (1988)

14. Hamilton, J.: Service value networks: Value, performance and strategy for the services industry. Journal of Systems Science and Systems Engineering 13(4), 469-489 (2004)

15. Kotler, P., Keller, K.: Marketing Management. Prentice Hall, Englewood Cliffs (2006)

16. Lam, S.Y., Shankar, V., Erramilli, M.K., Murthy, B.: Customer value, satisfaction, loyalty, and switching costs: An illustration from a business-to-business service context. Academy of Marketing Science 32, 293-311 (2004)

17. Legarreta, J.M.B., Miguel, C.E.: Collaborative relationship bundling: a new angle on services marketing. International Journal of Service Industry Management 15, 264-283 (2004)

18. Nakamura, K., Aoyama, M.: Value-Based Dynamic Composition of Web Services. In: AsiaPacific Software Engineering Conference (2006)

19. Premkumar, G.: Alternate distribution strategies for digital music. Communications of the ACM 46, 89-95 (2003)

20. Razo-Zapata, I.S., Chmielowiec, A., Gordijn, J., Van Steen, M., De Leenheer, P.: Generating value models using skeletal design techniques. In: 5th International BUSITAL Workshop (2010)

21. da Silva, G.E., Pires, L.F., van Sinderen, M.: Towards runtime discovery, selection and composition of semantic services. Comput. Commun. 34(2), 159-168 (2011)

22. Sivashanmugam, K., Miller, J.A., Sheth, A.P., Verma, K.: Framework for Semantic Web Process Composition. Int. J. Electron. Commerce 9(2), 71-106 (2005)

23. Traverso, P., Pistore, M.: Automated Composition of Semantic Web Services into Executable Processes, pp. 380-394. Springer, Heidelberg (2004)

24. Zlatev, Z.: Goal-oriented design of value and process models from patterns. $\mathrm{PhD}$ thesis, Twente University (2007) 